\title{
Management of patients with complicated intra- abdominal infections: three crucial steps
}

\begin{abstract}
Editorial
Major milestones in surgery have included avoidance of pain with the use of anesthesia, antisepsis to prevent operative contamination, secure sutures to promote tissue integrity, and cautery to minimize bleeding. In the antibiotic era, surgical procedures for source control in abdominal sepsis have been complemented with drugs targeting persistent organisms after luminal inflammation, obstruction, or perforation. Intra-abdominal infections (IAIs) encompass a variety of pathological conditions, ranging from uncomplicated appendicitis to fecal peritonitis. ${ }^{1}$ IAIs are subcategorized in 2 groups: uncomplicated and complicated IAIs (cIAIs). In the event of an uncomplicated case of IAI, the infection involves a single organ and does not spread to the peritoneum. Patients with such infections can be treated with either surgical intervention or antibiotics. ${ }^{2}$ When the infection is effectively resolved by means of surgery, a 24-hour regimen of perioperative antibiotics is typically sufficient. Patients with uncomplicated intraabdominal infections, such as acute diverticulitis, acute cholecystitis, and acute appendicitis, may be treated non-operatively by means of antimicrobial therapy. The complicated IAI usually demands surgical or another form of source control in order to resolve it. It generally arises from one of the hollow viscous organs, such as the colon or the appendix. These infections are the second most common cause of mortality in the ICU.
\end{abstract}

\section{First step for ciais: source control}

Source control is paramount in the patient with septic shock, and it should not be delayed until the patient is completely stable physiologically because that is not possible in most patients. What is needed is judicious resuscitation and making sure the anesthesiologist and others in the OR are prepared to continue that, and getting the patient to the OR for a source control procedure, even if only an abbreviated one (damage control). Early control of the septic source is mandatory and can be achieved by operative and non-operative means. The safety and efficacy of ultrasound-and CT-guided percutaneous drainage of abdominal abscesses has been documented in patients with appendiceal and diverticular abscesses. Percutaneous image-guided drainage may also be used to address cases of advanced acute cholecystitis. Two major clinical factors predicting failure of source control are delay in the initial intervention $(>24 \mathrm{~h})$ and inability to achieve adequate debridement or control of drainage.

\section{Second step: bacteria involved}

Data from clinical trials for IAIs showed that by far the most common invasive pathogen is Escherichia coli, followed by Klebsiella which accounts for about $10 \%$ of and Pseudomonas accounts for about $5 \%$ to $8 \%$ of community-acquired IAIs. Other organisms are so infrequent that we do not empirically treat for them. Another point is that the complexity of the infecting flora causes a very different type of infection than is seen, for example, with a lung infection, which is typically monomicrobial. In polymicrobial infections, including most notably E. coli, Bacteroides Fragilis, and in some cases, Streptococcus, it is very clear that the organisms interact with each other and cause infections at much lower densities and
Volume 3 Issue 2 - 2016

Vasileios Papadopoulos

Aristotle University of Thessaloniki, Greece

Correspondence: Vasileios Papadopoulos, Professor of Surgery, Medical School, Aristotle University of Thessaloniki, Greece, Email bnpap@med.auth.gr

Received: May 28, 2016 | Published: May 30, 2016

much different patterns compared with monomicrobial infections. And, these organisms cause local necrosis and thrombosis of blood vessels, allowing the organisms to spread with very low inocula along facial and even subcutaneous facial planes. Other issues to consider are if the patient has a balanced flora (eubiosis) or an imbalanced one (dysbiosis); food intake affecting the intestinal barrier function; use of drugs that reduce gastric acidity (achlorhydria) have effect on increasing strains such as E. Coli and Bacteroides frag.

\section{Third step: empiric antibiotic therapy: which drugs? when? how long?}

Antimicrobial therapy should be initiated once a patient receives a diagnosis of an intra-abdominal infection or once such an infection is considered likely. For most patients, the time to begin antibiotic therapy is when you suspect an infection; even patients with appendicitis should undergo early treatment. It removes the risk for progression of their infection while they wait to go to the OR, and in some cases operation may not even be necessary. The general rule is that it should be given within approximately 4hours in patients who are not in septic shock. In addition, antibiotic therapy for a patient undergoing operation has a role in wound prophylaxis, as with any surgical procedure. ${ }^{3}$ This may require, depending on the agent used (eg, an agent with a short half-life), additional doses just before the initiation of the procedure. Regarding the selection of accurate empiric antibiotic therapy it may be 2 or 3 days before we get accurate microbiologic data. A general consideration is the origin of the infection and the severity of the infection; for example, a perforated duodenal ulcer is usually associated with gram-positive organisms with a relatively low inoculum. Patient risk factors for resistance are also important.

In most mild or moderate severity infections may be administered as single agent cefoxitin, ertapenem, moxifloxacin, or tigecycline. As combination regiment typically are used a first- or second-generation cephalosporin, or even ceftriaxone with metronidazole for a lot of the mild-to-moderate infections, such as a perforated appendicitis. Carbapenem (piperacillin-tazobactam and then combination regimens with ceftazidime or cefepime) can be used for high gravity infections, patients with cIAI and high severity scores. Typically these are people who go to the ICU. In this group is recommended ceftazidime with metronidazole or clindamycin. Ceftazidime does not have good grampositive activity; on the contrary Cefepime is much better against 
gram-positive organisms. If patient is allergic to beta-lactam then must be administered a quinolone (Moxifloxacin) or combination quinolone (Ciprofloxacin or levofloxacin) with metronidazole. ${ }^{4}$

Recent STOP IT trial, which is the first solid randomized trial of stopping antibiotic therapy early, changed the everyday practice. Antimicrobial therapy is limited to 4days, as prolonged antimicrobial therapy provides no benefit in adult patients who have adequate source control. The appropriate duration of postsurgical antibiotic therapy has been unclear in cIAIs. However, if safe, shorter courses would be desirable to minimize antibiotic resistance, drug-related adverse events, and costs.

\section{Acknowledgements}

\section{None.}

\section{Conflict of interest}

The author declares no conflict of interest.

\section{References}

1. Singer M, Deutschman CS, Seymour CW, et al. Caring for the critically ill patient the third international consensus definitions for sepsis and septic shock (Sepsis-3). JAMA. 2016;315(8):801-810.

2. Wentzel RP, Edmond MB. Antibiotics for abdominal sepsis. $N$ Eng J Med. 2015;372(21);2062-2063.

3. Solomkin JS, Mazuski JE, Bradley JS, et al. Diagnosis and management of complicated intra-abdominal infection in adults and children: Guidelines by the surgical infection society and the infectious diseases society of America. Clin Infect Dis. 2010;50(2):133-164.

4. Sartelli M, Viale P, Catena F, et al. WSES guidelines for management of intra-abdominal infections. World J Emerg Surg. 2013;8(1):3. 